\title{
Organizational culture and climate factors impacting forensic interviewers' experiences of vicarious
}

\section{trauma}

\begin{abstract}
Hazardous" is not an adjective that comes to mind when describing children's advocacy centers. The reality, however, is that those who work within these organizations, such as forensic interviewing professionals, often experience symptomology similar to those who receive services. ${ }^{1,2}$ Data was collected utilizing an emergent, phenomenological design to better understand the context within which forensic interviewers experience, and are impacted by secondary trauma. A purposeful, representative sample $(n=9)$ of forensic interviewing professionals participated in semi-structured interviews regarding their perceptions of vicarious trauma as it relates to their job as forensic interviewers. Analysis of the data was rooted in constructivist grounded theory. ${ }^{3}$ Findings of this study point to the importance of considering the impact of organizational context and systems on how forensic interviewers' are impacted by vicarious trauma.
\end{abstract}

Keywords: vicarious trauma, forensic interview, child sexual abuse, trauma, organizational culture, organizational climate
Volume 4 Issue 4 - 2017

\author{
Amber R McDonald,' Jennifer Middleton, ${ }^{2}$ \\ Dawn Matera Bassett, ${ }^{3}$ Lesley Harris ${ }^{2}$ \\ 'University of Denver, Graduate School of Social Work, \\ Colorado \\ ${ }^{2}$ University of Louisville, Kent School of Social Work, Kentucky \\ ${ }^{3}$ Metropolitan State University of Denver, Colorado
}

Correspondence: Amber R McDonald, University of Denver, Graduate School of Social Work, 2 I 48 S. High Street, Denver, CO 80208, Colorado, Email mcdonald.amber.r@gmail.com

Received: October 15, 2016 | Published: April 12, 2017

\section{Introduction}

"Hazardous" is not an adjective that comes to mind when describing children's advocacy centers. The reality, however, is that those who work within these organizations, such as forensic interviewing professionals (FIs), often experience symptomology similar to those who receive services. ${ }^{1,2}$ FIs are tasked with talking to children who are victims or witnesses to a criminal act at the front end of an investigation involving a child who has been a victim or witness to a crime. The literature is imprecise in its articulation of terminology when describing the experiences of professionals who work with survivors of trauma (e.g. cancer patients, survivors of domestic violence, sexual violence, child abuse, etc.). ${ }^{4}$ Terms such as: vicarious trauma, ${ }^{1,5-7}$ secondary trauma, ${ }^{1,2,8,9}$ compassion fatigue, ${ }^{8}$ disengagement, ${ }^{2}$ and burnout ${ }^{2}$ are all used to describe how professionals embody the traumatic experiences of their clients. This is explained as a process of the professional being vicariously exposed to the traumatic experiences of their clients, which results in the manifestations of their own trauma symptomology. ${ }^{10}$ Vicarious trauma and secondary trauma are the predominant areas of focus for scholars interested in the well-being of trauma professionals. Vicarious trauma is defined as profound changes in an individual's perception of self which results in disruptions of cognitive schemas related to identity, memory and belief systems. ${ }^{11}$ This construct is different than secondary trauma which seeks to identify stressors related to helping or wanting to help a traumatized person. ${ }^{7}$ Very broadly, there has been minimal research conducted with FIs regarding their trauma experiences (vicarious trauma or secondary trauma) associated with their work. What is available is exclusively focused on disengagement and secondary trauma. Findings from these preliminary studies, indicate that while tenure and interviewing load are related to higher levels of disengagement ${ }^{2}$ among interviewers, ${ }^{1}$ external social support (e.g., support from family and friends) and internal job support (e.g., support from supervisors, administrators, and colleagues) are important predictors of interviewer's levels of secondary traumatic stress. ${ }^{1}$ By the same token, research suggests that vicarious trauma has a direct effect on turnover among professionals who work in fields closely related to forensic interviewing. ${ }^{12}$ Due to the dearth of research available, and the implications high annual turnover rates have for the organizations that employ FIs (e.g., fiscal implications, impact on quality of interviews, client outcomes), this research study sought to identify what factors of organizational culture and/or climate impact FIs experiences of vicarious trauma.

\section{Literature review}

Vicarious trauma and the subsequent intent to leave is a recognized workforce problem within organizations that provide services to traumatized clients. With agencies experiencing annual turnover rates between $20-60 \%$, employers have a compelling need to address these difficulties. ${ }^{13}$ The complexity of employee retention is further exacerbated because $50 \%$ of the clinicians with advanced professional degrees are leaving the field within two years ${ }^{13}$ yet it takes a minimum of two years for a clinician to develop the skills to become effective and autonomous. ${ }^{14}$ To address this workforce issue, researchers and organizations are exploring factors which influence retention. One important occupational construct associated with job stress and turnover is vicarious trauma. ${ }^{6,15}$ Theorists differentiate vicarious trauma from other processes related to professional's experiences of their client's trauma (e.g., compassion fatigue, secondary traumatic stress) in that vicarious trauma is characterized by a shift in intrapersonal processes (i.e., changes in trust, feelings of control, issues of intimacy, esteem needs, safety concerns, and intrusive imagery) as a result of working with traumatized clients. ${ }^{11}$ Unlike compassion fatigue (a reduced capacity or interest in being empathic or bearing the suffering of clients which is a consequence of knowing about a traumatizing event experienced by a person; ${ }^{16}$ or secondary traumatic stress (an acute stress reaction to a secondary traumatic event which mimics post-traumatic stress disorder; PTSD) $)^{8,9,17}$ vicarious trauma results in a cognitive shift which involves "profound changes in the core 
aspects of the professional's self and... may involve disruptions in the cognitive schemas" of helping professionals' "identity, memory system, and belief system". ${ }^{11}$ As we have become familiar with the various constructs associated with organizational and workforce well-being, researchers are now focusing on the nature of these occupational stress phenomena.

Research has found that there are intrapersonal and organizational factors that increase clinicians' risks for experiencing vicarious trauma in their work. ${ }^{5,15,18}$ One study of 188 self-identified trauma therapists found that newer and younger clinicians reported a greater level of distress in their work. ${ }^{7}$ New clinicians may have anxiety about their professional viability and this anxiety increases their risk for occupational distress. However, being a new clinician is not the only risk factor for work related stress. In a study of 152 clinicians, researchers found that there was a positive correlation between stress reactions and large caseloads, increased contact with clients and long working hours. ${ }^{19}$ Clinicians with a history of personal trauma have also been found to be at a greater risk for traumatization. While future research is needed to fully understand the relationship between personal trauma and workplace traumatization, Pearlman et al., ${ }^{5}$ proposed that the clinician's beliefs about their own safety and beliefs about intimacy with others may contribute to their levels of distress. Other factors associated with increased risk are working with sexually traumatized clients, having experience in the field, and being female. ${ }^{20}$ In a recent study of 1,192 child welfare professionals who work directly with traumatized clients, Middleton et al., ${ }^{12}$ found that there was a significant relationship between experiencing vicarious trauma and the professional's intent to leave their jobs. While this study was not conducted with FIs, it does raise the issue of whether FIs who interview traumatized clients will manifest vicarious trauma similarly.

Research on issues associated with retention among FIs has just started to occur over the past 10 years, and as mentioned previously, studies have not examined the construct of vicarious trauma specifically. The research has predominantly focused on secondary traumatic stress which, when measured empirically, only includes symptoms experienced in the past seven days. ${ }^{21}$ However, it is well documented that social workers (overall) who treat clients with trauma related issues, are reporting active and persistent symptomology related to a diagnosis of Post-Traumatic Stress Disorder. ${ }^{21}$ Further, professionals who specialize in violent clinical populations (i.e., those who work in the areas of child sexual abuse, child abuse, and torture) are scoring higher on the Traumatic Stress Institute (TSI) Belief scale $^{5}$ than their peers in other specialties. ${ }^{22}$ These same specialized professionals are reporting more disruptions in their perceptions of self-safety and devaluing others at a rate higher than their clinical peers (Cunningham, 2004). This information signifies that trauma professionals, who closely parallel the role of FIs, are struggling with the material they are exposed to in their professional role far beyond the symptomology of secondary traumatic stress (e.g., intrusion, arousal, avoidance). In an effort to enhance knowledge about how occupational factors effect forensic interviewers, this qualitative study explored how organizational culture and climate factors impact FIs' experiences of vicarious trauma.

\section{Theoretical framework}

Constructivist Self-Development Theory (CDST) is the theoretical lens applied to the data in this study. The underlying premise of this theory is that human beings construct their own personal realities through the development of complex cognitive structures which are used to interpret events. ${ }^{11}$ More specifically, CSDT recognizes the influence of an individual's history and/or personality on their perception of traumatic events. Through this account, the application of this theory provides a glimpse into the individual perceptions of FIs' traumatic experience in their social (organizations) and cultural contexts. ${ }^{23}$ Attending to the uniqueness of FIs' experiences of vicarious trauma from an organizational and cultural context provides an opportunity for organizational leadership teams within child advocacy centers to attract and respond to healthy and competent FIs.

\section{Methods}

\section{Study design}

This qualitative study involved in-depth interviews with a purposive sample of nine forensic interviewing professionals employed and practicing in a large, western state. Data collection took place as part of a larger phenomenological study examining the impact of vicarious trauma among forensic interviewing professionals. The objective of the current study was to understand how organizational culture and climate factors impact forensic interviewers' experiences of vicarious trauma. The sampling frame for the study included all 12 experienced FIs who were working in the state during the year that this study was conducted. Nine out of the total 12 forensic interviewing professionals who were working in the state agreed to be interviewed (75\% response rate). Inclusion criteria did not explicitly state that participants had to have experienced vicarious trauma to participate in the study. At the conclusion of interviews, participants received a five-dollar gift card to a retail store. All study policies and procedures were approved by the institutional review board of [blinded for review].

Most of the participants were women (78\%), Euro-American/ Caucasian (77.8\%), and five of the participants had completed a fouryear degree, whereas the other four had completed a Master's degree. In regards to professional experience, five of the forensic interviewers indicated working at child advocacy centers (CAC) $(55.6 \%)$, two at law enforcement (LE) agencies $(22.2 \%)$, and two at departments of human services (DHS/DSS) (22.2\%). The majority of the participants reported being very satisfied $(56 \%)$ with their jobs. Other participants reported being satisfied $(33 \%)$ or very unsatisfied $(11 \%)$ with their jobs. In regards to years of experience in the "field," participants reported an average of 9.5 years, with a range of 2.5 to 18 years. In regards to forensic interviewing specifically, the participants reported a mean of 5.5 years of experience, with a range of 0.5 to 18 years. All of the participants reported that they had experienced vicarious trauma symptoms, which were indicated by physical symptoms, emotional symptoms, behavioral symptoms, work related issues, interpersonal problems, and/or professional efficacy such as a decrease in concern and esteem for clients; $; 1,24-28$ Perry ${ }^{29}$ Researchers did not inquire about historical mental health and/or trauma of participants prior to the commencement of the study.

\section{Data collection and analysis}

Interviews were conducted between February and March 2008. All of the interviews took place in person. In-depth qualitative interviewing was used to facilitate an understanding regarding how FIs experience vicarious trauma, and how their interpretations are influenced by the context within which they work. ${ }^{30,31}$ On average, the research interviews lasted 2.5 hours and were digitally recorded, professionally transcribed, and checked for accuracy against original audio files. Some of the interview questions included: "How would you define or describe vicarious trauma?" and, "As a professional who conducts forensic interviews with children, how are you impacted 
by vicarious trauma?" (Prompts: physically, mentally, emotionally, socially, professional effectiveness). Transcripts were managed in ATLAS. ti, a software program that facilitates qualitative data analysis and management. Personal identifiers were removed from all transcripts. In order to increase the rigor of the coding process, as well as minimize potential researcher bias, reflexive memoing ${ }^{3}$ was conducted at each stage of the coding process with the lead-researcher and the research team. This technique provided a venue for the leadresearcher to identify personal bias and create transparency with the research team as it relates to the analysis of the data.

The data was analyzed using a constructivist grounded theory approach with open coding, focused coding, and axial coding strategies. ${ }^{3}$ Each transcript was initially read in its entirety. Beginning with open coding, the researcher highlighted key phrases that related to the individual's organizational culture and climate and their experiences within the multi-disciplinary teams in which they engage, in the transcripts and paraphrased them into condensed open code, capturing the essence of each phrase. After $20 \%$ of the transcripts had been coded, the lead-researcher met with the original researchers to ensure coding consistency. Using the family network manager in Atlas. ti, the researchers used open codes to create focused codes, or clusters of similar or related codes, and then continued to meet regularly to discuss the content of each transcript to identify emerging categories. After completing open and focused coding, a preliminary codebook that listed the most recurrent and initial codes was developed in the family network manager of Atlas.ti. The remaining interview transcripts were analyzed using the codes established in the codebook. The codebook consisted of a hierarchy, which included three code families that each contained two sub-codes (internal and external factors), and 12 child codes with definitions.

After the codebook was established, the lead-researcher coded all nine interviews and conferred with the other researchers during and after the coding process. In these meetings, the researchers discussed coding patterns and resolved disagreements related to code definitions. This iterative process helped enhance trustworthiness of the analysis and led to a well-defined codebook. ${ }^{32}$ The dependability of the findings in the study was established by the transparent coding process, and the regular meetings of the researcher team, which involved building consensus and peer debriefing. ${ }^{33}$ To visually present the data for further analysis, the codes were used to create data-family matrices. Analysis within and across cases and coding categories of the families led to the construction of several overriding themes and axial codes which related to organizational and climate factors that impacted participants' experiences of vicarious trauma. At the axial level of coding, two researchers worked together by comparing data and interviews for variations and nuances to create descriptive categories within each theme. From these conversations, subcategories that described the properties and dimensions of the categories were articulated. ${ }^{3}$ Throughout the analysis process, researchers focused on using the data to describe participants' understandings of how their organizational climate impacted their experiences of vicarious trauma. Additionally, the authors maintained memos related to analytic decisions, consulted with other members of the research team, and discussed the relationships among codes that emerged from the data. ${ }^{34,35}$ In order to address the issue of credibility and trustworthiness. ${ }^{36,37}$ the researchers employed member checking techniques and attended to prolonged engagement. In regards to member checking, researchers agreed to initiate it in the interview itself, similar to getting clarification from participants. The researchers also decided to employ member checking techniques after the initial data analysis phase was complete, in which findings were presented back to the participants on an individual basis.

\section{Findings}

Three themes were identified: Isolation, Organizational Factors, and Norms of Self-Sacrifice. A conceptual map provided below (Figure 1) provides a visual representation of the coding process (axial coding, identifying properties and dimensions of the categories, and the creation of the larger codes; Charmaz et al., ${ }^{3}$ as described above. Isolation encompasses the high levels of seclusion forensic interviewing professionals experience within themselves and in the systems with which they engage. Organizational Factors is defined as the positive and negative aspects of organizational context that FIs experience as a result of working within varying types of organizations. Self-sacrifice represents the deleterious choices made by forensic interviewers that are motivated by their love and compassion for the children they serve.

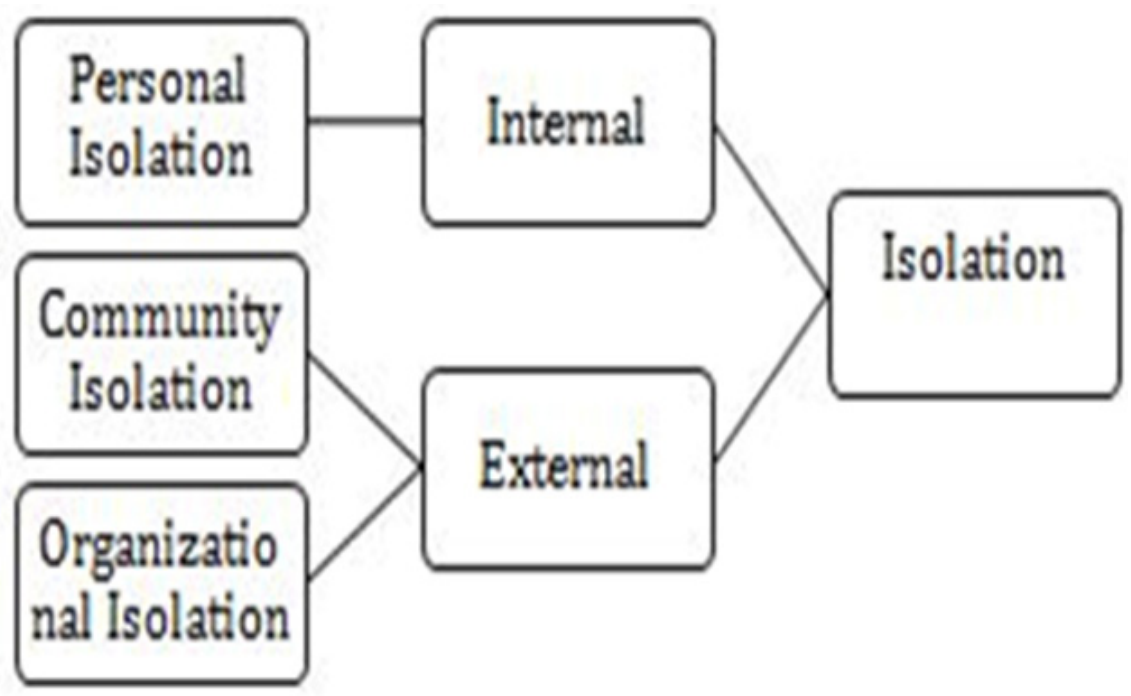




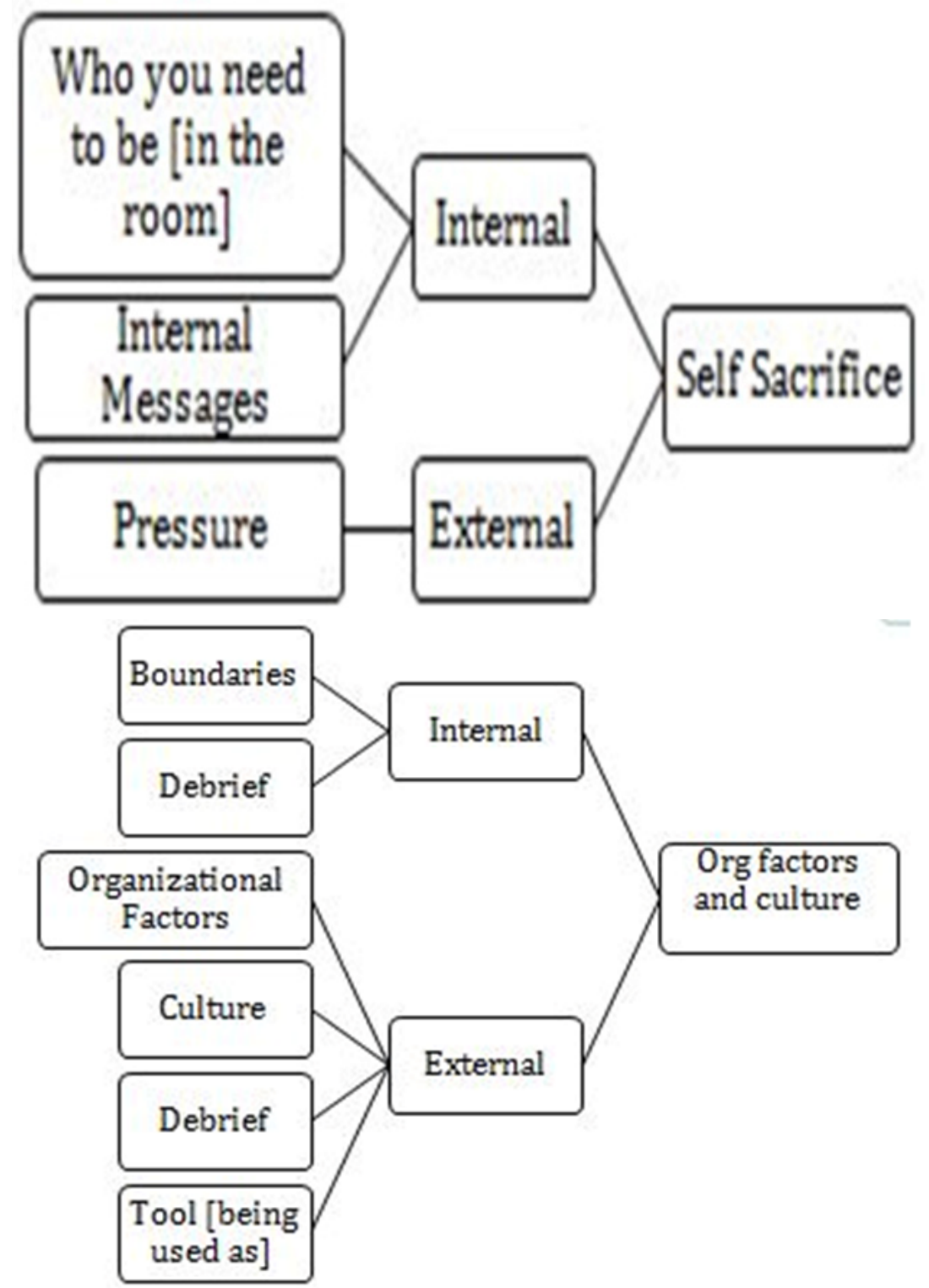

Figure I Visual representation of the coding process.

\section{Isolation}

As a forensic interviewer specializing in the area of child sexual abuse (CSA), participants report an overwhelming feeling of isolation and quotes support this theme. Participants report experiencing isolation on an individual level, within their professional organization, and in their community. Many mention that they do not talk about their work to others in their communities or social circles they engage in because of the immense level of discomfort that arises. The following quote illustrates a participant's uncomfortable experience with sharing what she does for a living with those outside the profession, “...when it's somebody who says, 'oh, that's gotta be so hard,' or everybody gives you the same face. Oh, like this disgusting face like, 'oh my God, why do you do that work?' And it's just always an uncomfortable spot to be in" (participant number 201). Another participant reflects on the large piece of her life that she cannot share with others despite its profound impact on her: "There's a large part of your life that you can't talk about. Um, that is a really huge part of your life that is affecting you" (participant number 203). Others talk about how specializing in this type of work deems you as "other" with colleagues within their same organization, "Nobody wanted to come to that unit, talk about it. 'How do you guys do that?' so you felt really isolated. Only we understand..." (participant number 302). Those who were not part of larger teams (e.g., law enforcement or social services) mentioned that their isolation was rooted in being the only professional within their organization that understood or knew what it felt like to forensically interview a child. Interviewers who worked at centers with other forensic interviewers mentioned that having a colleague who did the same job on site with them lowered their feelings associated with vicarious trauma.

\section{Organizational factors}

Flexibility, understanding and attentiveness on behalf of supervisors and upper-management, in all types of organizations, were positive influences in mitigating levels of vicarious trauma. 
Specifically, participants expressed that supervisors who encouraged coming in late after a long day, taking off an afternoon because they could sense irritability on behalf of the interviewer, and effectively managing the number of interviews an interviewer was conducting were especially helpful. Participants expressed frustration when individuals in leadership roles were unaware of what their day-to day-job looked like and failed to understand the demanding nature of the job. A participant describes her frustration with her Department Director: "The Director, who is the head of the system, has no idea of the level of trauma. Um, and that has been frustrating in some ways when it comes to support ...I think if you have appreciation for the vicarious trauma, and the increase in burn out, then as an administrator, you will be more supportive of getting people the help that they need. But there's a disconnect there..." (participant number 202).

Participants were split about the benefits and hindrances of working in different types of organizational systems (e.g., police department/social service agency vs. child advocacy center/small non-profit). Most highlighted that working in larger organizational systems had the benefit of having a team who understood the dayto-day work that they were doing. The participant below articulates the perks associated with having colleagues who were fully aware of the intricacies of the cases she was engaged in when she was a detective versus a more isolative experience in her current role as a forensic interviewer at a child advocacy center, "I'm in a different position here than, at work, than when I was a detective; "cause we worked together, and we knew each other's cases, and it was easier" (participant number 203). But, many mentioned that the pressure of having to handle the case in its entirety, which is the responsibility of those working in larger organizational systems, was much more stressful and traumatic than only meeting the child on that one occasion, conducting the forensic interview, and not following the case in its entirety (i.e., working as a forensic interviewer at a child advocacy center). Being exposed to slivers of the case and not having to manage families and larger systems on a daily basis was a positive aspect provided by work within child advocacy center settings. Below is a quote from a participant who is describing the benefit of being a part of a child advocacy center, a smaller organization with limited involvement with families, "I'm just a piece, I'm an investigative tool they use and that's how they use it" (participant number 302).

In contrast, $44 \%$ of the participants described how the brief role in a case appeared to diminish the level of individual importance of the interviewer to the larger team. While forensic interviewers who were only responsible for interviewing and not the case in its entirety had the benefit of minimized trauma, participants articulated feeling as though their "person" or sense of agency as it related to their position within the larger MDT was less valuable. This is most clearly demonstrated by a participant who talked about being with her terminally ill mother who was on her "death bed" and having an attorney call her from her MDT to tell her that she must promptly leave her mother's bedside and get to court or else she would be held in contempt of court. This type of perceived betrayal by a member of her MDT had a lasting, negative effect on the interviewer, who tearfully recalled the event.

\section{Self-sacrifice}

Because of the profound importance of their job and the vulnerable nature of the children they encounter, the participants appeared to create and respond to a culture of self-sacrifice. Self-sacrifice, defined as the decision to give up one's commitment to self-care or balance between their personal and professional's lives, was a common theme described by all of the participants. Participant number 202 describes this, "..the survivors guilt of taking two weeks off, and knowing that whoever's coming in after me is gonna have to do my job. And, the guilt of unprotected children out there. Um, it took me two weeks to give myself permission to take care of me." Some talked about pushing through despite being completely burned out and others mentioned how everything in their world is pushed aside, regardless of its importance, to be able to put their best foot forward for the child they were about to interview, "No matter what your problems are here, your whole life, as soon as you leave these doors it's a whole nother world. You have to forget about what's going on and focus on their needs, especially the ages that they are" (participant number 302). While these participants have a distinct ability to identify when they are on the edge, they need some validation (sometimes from themselves) that it is okay to care for themselves, "I've given myself a lot more permission to work less" (participant number 205).

\section{Discussion}

Findings of this study point to the importance of considering the impact of organizational context and systems on how forensic interviewers' are impacted by vicarious trauma. However, several limitations exist and must be considered when interpreting the findings. While the sample represents a majority of FIs in the state, it only represents one western state. In addition, the sample consists predominantly of Euro-American women. While this is probably representative of FIs around the country, it is important to include other groups to better understand the phenomenon. Furthermore, while reflexive memoing was utilized to identify and bracket personal biases and create transparency with the research team as it relates to analysis, it is important to consider how effectively the team's biases were bracketed. Also, not all participants in this study were solely forensic interviewers (e.g., $100 \%$ of their job responsibility is to interview children). A subset of the participants had additional job responsibilities; therefore, their experiences may be different than those who are only responsible for interviewing. However, one could argue that this variation in job roles and responsibilities helped to illuminate differences across varying organizational contexts. The research team worked to capture and articulate the differences in experiences, as applicable, in the themes derived. Finally, participants were not interviewed about current or past trauma experiences. Thereby, findings of this study do not account for the potential of historical trauma impact on behalf of the individual forensic interviewing professional, ${ }^{38}$ and their feelings of vicarious traumatic stress.

\section{Implications}

Despite these limitations, this study offers several important implications regarding theory, research, and practice in the field of forensic interviewing. In regards to theory, the findings seem to support Pearlman and McCann (1995) conceptualization of the impact of vicarious trauma within the context of Constructivist Self Development Theory (CSDT). CSDT perceives individuals' adaptations to trauma "as interactions between their own personalities (defensive styles, psychological needs, coping styles) and salient aspects of the traumatic events, all in the context of social and cultural variables that shape psychological responses". ${ }^{7}$ As described in the results section, specific organizational culture and climate factors such as the MDT and leadership play an important role in how FIs experience and make meaning of their vicarious trauma.

Not only does the current study illuminate the unique experiences of FIs regarding vicarious trauma, the study also has organizational and policy implications for child advocacy centers and the leadership of those agencies. Findings highlight the need for additional support 
for FIs, particularly those who work in more isolative environments and/or are the only person performing their interviewing role in their agency. In addition, there are important considerations in regards to retention practices for FIs. While current practices focus on the enhancing coping strategies among the interviewers themselves, the findings indicate a need to consider the impact of the organizational context on the interviewer's ability to cope. Organizations need to provide training regarding trauma-responsive practice for MDT members and key leadership within child advocacy centers and other organizations. A team-based, trauma-informed organizational change intervention, such as The Sanctuary Model ${ }^{\circledR}$, could help FIs feel supported and could promote a safe, empowering, and resilient organizational climate. The Sanctuary Model is an evidence supported and trauma-informed theoretical model that relies on a structured methodology for creating or changing an organizational culture. ${ }^{39}$ But first, the study could be expanded to include a broader sample of forensic interviewing professionals. In addition, the conceptual model developed as an outcome of the current qualitative study should be tested further. Using quantitative research methods and a national sample of FIs, the primary themes of isolation (including personal, professional, and community domains), self-sacrifice, and organizational environment (e.g., culture, climate, and leadership) could be operationally defined and measured using psychometrically valid scales to examine the relationships between the constructs. It would be important to determine which organizational climate factors are negatively associated with vicarious trauma in order to inform targeted interventions for the organizations that house FIs. ${ }^{40-43}$ Considering the important role FIs play in child sex abuse investigations and prosecutions, and considering the organizational cost of losing a seasoned FI to vicarious trauma, interventions aimed at retaining healthy and competent FIs are worthy of additional research.

\section{Acknowledgments}

None.

\section{Conflicts of interest}

The author declares there are no conflicts of interest.

\section{References}

1. Bonach K, Heckert A. Predictors of secondary traumatic stress among children's advocacy center forensic interviewers. J Child Sex Abus. 2012;21(3):295-314.

2. Perron BE, Hiltz BS. Burnout and secondary trauma among forensic interviewers of abused children. Child and Adolescent Social Work Journal. 2006;23(2):216-234.

3. Charmaz K. Constructing grounded theory: A practical guide through qualitative analysis. 2nd edn. 2006;1-224.

4. Knight C. Indirect trauma: Implications for self-care, supervision, the organization, and the academic institution. The Clinical Supervisor. 2013;32(2):224-243.

5. McCann I, Pearlman L. Vicarious traumatization: A framework for understanding the psychological effects of working with victims. Journal of Traumatic Stress. 1990;3(1):131-149.

6. Pearlman L, Saakvitne KW. Trauma and the therapist: Counter transference and vicarious traumatization in psychotherapy with incest survivors. 1995; 1-451.

7. Pearlman LA, MacIan P. Vicarious traumatization: an empirical study of the effects of trauma work on trauma therapists. Journal of Professional Psychology: Research and Practice. 1995;26(6):558-565.
8. Figley CR. Compassion fatigue: Coping with secondary traumatic stress disorder in those who treat the traumatized. Routledge, USA. 1995;1292.

9. Stamm BH. Secondary traumatic stress: Self-care issues for clinicians, researchers, and educators. Sidran Press, USA. 1995;1-332.

10. Sommer CA, Cox JA. Elements of supervision in sexual violence counselors' narratives: A qualitative analysis. Counselor Education and Supervision. 2005;45(2):119-134.

11. Trippany RL, White Kress VE, Wilcoxon SA. Preventing vicarious trauma: What counselors should know when working with trauma survivors. Journal of Counseling \& Development. 2004;82(1):31-37.

12. Middleton JS, Potter CC. Relationship between vicarious traumatization and turnover among child welfare professionals. Journal of Public Child Welfare. 2015;9(2):195-216.

13. Paris M, Hoge MA. Burnout in the mental health workforce: A review. $J$ Behav Health Serv Res. 2009;37(4):519-528.

14. Auerbach C, McGowan BG, Ausberger A, et al. Differential factors influencing public and voluntary child welfare workers' intention to leave. Children and Youth Services Review. 2010;32(10):1396-1402.

15. Canfield J. Secondary traumatization, burnout, and vicarious traumatization: A review of the literature as it relates to therapists who treat trauma. Smith College Studies in Social Work. 2005;75(2):81-101.

16. Adams RE, Boscarino JA, Figley CR. Compassion fatigue and psychological distress among social workers: A validation study. Am J Orthopsychiatry. 2006;76(1):103-108.

17. Phipps AB, Byrne MK. Brief interventions for secondary trauma: review and recommendations. Stress and Health. 2003;19(3):139-147.

18. Dunkley J, Whelan TA. Vicarious traumatization: current status and future directions. British Journal of Guidance \& Counseling. 2006;34(1):107116.

19. Devilly GJ, Wright R, Varker T. Vicarious trauma, secondary traumatic stress or simply burnout? Effect of trauma therapy on mental health professionals. Aust N Z J Psychiatry. 2009;43(4):373-385.

20. Woodard MT, Cornille T. The trauma of working with traumatized children. In: Figley CR, editor. Treating compassion fatigue. BrunnerRoutledge, USA. 2002;39-55.

21. Bride BE. Prevalence of secondary traumatic stress among social workers. Soc Work. 2007;52(1):63-70.

22. Bober T, Regehr C. Strategies for reducing secondary or vicarious trauma: Do they work? Brief Treatment and Crisis Intervention. 2006;6(1):1-9.

23. Saakvitne KW, Tennen H, Affleck G. Exploring thriving in the context of clinical trauma theory: Constructivist self-development theory. Journal of Social Issues. 1998;54(2):279-299.

24. Bell H, Kulkarni S, Dalton L. Organizational prevention of vicarious trauma. Families in Society. The Journal of Contemporary Social Services. 2003;84(4):463-470.

25. Cherniss C. Resolving the "mixing and matching" problem: A view from the organizational perspective. In Tolan $\mathrm{P}$, Keys $\mathrm{C}$, editors. Researching community psychology: Issues of theory and methods. American Psychological Association, USA. 1990;134-138.

26. Clemans SE. Recognizing vicarious traumatization: a single session group model for trauma workers. Social Work with Groups. 2004;27(23):55-74.

27. Dane B. Duty to inform: Preparing social work students to understand vicarious traumatization. Journal of Teaching in Social Work. 2002;22(34):3-20. 
28. Dane B. Child welfare workers: An innovative approach for interacting with secondary trauma. Journal of Social Work Education. 2000;36(1):2738.

29. Salston M, Figley CR. Secondary traumatic stress effects of working with survivors of criminal victimization. Journal of traumatic stress. 2003;16(2):167-174.

30. Blumer H. Symbolic interactionism: Perspective and method. Englewood Cliffs, Prentice-Hall, USA. 1969;1-224.

31. Charon JM. Symbolic Interactionism: An Introduction, An Interpretation, 9th edn. Englewood Cliffs, USA. 2007;1-256.

32. MacQueen GK. Handbook for team-based qualitative research. AltaMira Press, USA. 2008;205-206.

33. Erlandson DA, Harris EL, Skipper BL, et al. Doing naturalistic inquiry: A guide to methods. USA. 1993;1-224.

34. Miles MB, Huberman AM. Qualitative data analysis: An expanded sourcebook. 1994.

35. Strauss A, Corbin J. Basics of qualitative research: Techniques and procedures for developing grounded theory. 3rd edn. 2008.
36. Guba EG, Lincoln YS. Competing paradigms in qualitative research. In: Denzin NK, Lincoln YS, editors. Handbook of Qualitative Research, 2nd edn. Sage, USA. 1998;105-117.

37. Lincoln YS, Guba EG. Naturalistic inquiry. 1985;1-416.

38. Van der Kolk BA, Roth S, Pelcovitz D, et al. Disorders of extreme stress: The empirical foundation of a complex adaptation to trauma. J Trauma Stress. 2005;18(5):389-399.

39. Esaki N, Benamati J, Yanosy S, et al. The Sanctuary Model: Theoretical framework. Families in Society: The Journal of Contemporary Social Services. 2013;94(2):87-95.

40. Creswell JW. Qualitative Inquiry and Research Design: Choosing Among Five Approaches, 3rd edn. 2013;1-472.

41. Jackson SF. A participatory group process to analyze qualitative data. Prog Community Health Partnersh. 2008;2(2):161-170.

42. Mishler EG. Research Interviewing: Context and Narrative. Harvard University Press, 1991;1-206.

43. Westhues A, Ochocka J, Jacobson N, et al. Developing theory from complexity: Reflections on a collaborative mixed method participatory action research study. Qual Health Res. 2008;18(5):701-717. 